\title{
Aplicación de arrays de anticuerpos en el estudio del cáncer vesical
}

\author{
Sánchez-Carbayo M.
}

Grupo de Marcadores Tumorales. Centro Nacional de Investigaciones Oncológicas. Madrid.

Actas Urol Esp. 2007;31(9):1082-1088

\section{RESUMEN}

APLICACIÓN DE ARRAYS DE ANTICUERPOS EN EL ESTUDIO DEL CÁNCER VESICAL

Los arrays de anticuerpos representan una tecnología de alto rendimiento muy versátil que permite la detección de múltiples proteínas simultáneamente. Al permitir cuantificar los niveles proteicos multiparamétricamente y analizar modificaciones post-translacionales, permiten mejorar la caracterización funcional de los procesos moleculares asociados con procesos neoplásicos. Más aun, la identificación de los patrones de expresión proteicos característicos de la progresión tumoral y de los distintos subtipos tumorales puede mejorar el manejo clínico del paciente con cáncer vesical y permitir en un futuro próximo el diseño de terapias adaptadas a la agresividad de cada tumor.

Palabras clave: Arrays de anticuerpos. Cáncer vesical. Proteómica.

\section{ABSTRACT}

USE OF ANTIBODIES ARRAYS IN THE STUDY OF BLADDER CANCER

Antibodies arrays represent a very versatile high-throughput technology that enables multiple protein detection simultaneously. It allows quantifying protein levels multiparametrically, and analyzing their post-translational modifications, thus improving the funtional characterization of molecular processes associated with neoplasic diseases. Moreover, the identification of protein expression patterns characteristic of tumor progression and of different tumor subtypes can improve the clinical management of patients with bladder cancer. In the near future, they may allow establishing tailored therapies according to the aggresiveness of each specific tumor.

Keywords: Antibody arrays. Bladder cancer. Proteomics.

\section{ARRAYS DE ANTICUERPOS EN EL CONTEXTO DE OTRAS METODOLOGÍAS DE PROTEOMICA}

El desarrollo de arrays de ADN ha permitido estudios de alto rendimiento cuantificando los niveles de RNA mensajero de diversos sistemas biológicos. El hecho de que el papel funcional de los mismos sea desempeñado por proteínas, unido a la falta de correlación exhaustiva entre los niveles de transcritos y proteínas han llevado al desarrollo de tecnologias de alto rendimiento para la determinación multiparamétrica de proteínas también. El aumento de técnicas de proteómica ha sido además favorecido por la relevancia biológica asociada a un mejor conocimiento en los niveles de expresión proteicos y sus modificaciones post-translacionales, y el hecho de que las proteinas representan también la mayor parte de dianas terapéuticas para ser desarrolladas farmacológicamente. Las técnicas de proteómica están encontrando una amplia aplicación en la determinación de las bases moleculares asociadas a cualquier enfermedad (proteómica funcional), las bases mecanísticas de acciones de fármacos así como su toxicidad (farmacoproteómica), y el desarrollo de nuevos marcadores específicos de cada enfermedad (proteómica clínica).

El proteoma de la célula cancerosa puede ser investigado usando distintas estrategias proteomicas que pueden ser guiadas o no. La termino- 
logía se refiere a si el proteoma a ser investigado se conoce previamente y es considerado en el diseño experimental (estrategia guiada). Los arrays de anticuerpos y proteínas representan plataformas proteómicas guiadas complementarias que disponen de ciertas ventajas como alta rentabilidad y reproducibilidad sobre tecnologias de proteómica no guiadas basadas en separaciones electroforéticas bidimensionales y de espectrometría de masas (Tabla 1).

Los arrays de anticuerpos consisten en portas de distintos materiales en los que los anticuerpos pueden inmovilizarse para capturar y cuantificar la presencia de proteínas específicas ${ }^{1}$. En los arrays de proteínas, pueden inmovilizarse péptidos, proteínas o complejos extractos proteicos. Una de las ventajas fundamentales de la tecnología de arrays de anticuerpos es su capacidad para medir cientos de proteínas simultáneamente usando pequeños volúmenes tanto de las muestras clínicas o biológicas a analizar, como de los anticuerpos generalmente caros. Esta plataforma proteómica guiada permite analizar diferencias cuantitativas en abundancia de proteínas y evaluar simultáneamente redes de señalización conocidas. Este abordaje permite diseños experimentales que responden a hipótesis específicas así como la interpretación biológica de los resultados obtenidos. Su versatilidad permite controlar y estimar su la reproducibilidad, calibración, precisión y sensibilidad de las mediciones multiparamétricas que se realizan. Sin embargo, el número de mediciones simultáneas que pueden realizarse a nivel práctico depende de la disponibilidad de anticuerpos con alta afinidad y especificidad disponibles para la caracterización de cada una de las proteínas diana a medir ${ }^{1-3}$.

\section{FORMATOS DE ARRAYS DE ANTICUERPOS}

La alta versatilidad en el diseño y formato de los arrays de anticuerpos ha conducido a múltiples clasificaciones y versiones de los mismos. El formato de los arrays de anticuerpos está relacionado con los procesos de marcaje e hibridación. La característica común de cada una de las versiones es que los anticuerpos a imprimir son utilizados como molécula de captura. El método de detección es complementario al diseño del formato del array de anticuerpos, al tipo de molécula de inmovilización y a la superficie donde son inmovilizadas. La inmovilización de los anticuerpos puede realizarse en una superficie plana del tipo de los soportes de filtro, microplacas o portaobjetos, dando lugar a los arrays planos ${ }^{1-4}$. Los arrays pueden ser en suspensión, de manera que superficies circulares pueden ser rodeadas por el anticuerpo de captura que se une a la proteína diana y son separadas e identificadas usando un sistema tipo citometría de flujo ${ }^{5,6}$. La innovación en las estrategias de inmovilización y detección de superficie ha conducido a un aumento en las tecnologías de arrays planos y en suspensión (Fig. 1).

Los principales tipos de arrays planares se clasifican en función del marcaje de los mismos, bien usando un único anticuerpo, o bien en arrays "sándwich", que usan dos anticuerpos para capturar la proteína diana ${ }^{1-4}$. Ambos tipos presentan ventajas e inconvenientes cuando se comparan entre sí. En los arrays basados en el marcaje con un anticuerpo, las proteínas son capturadas por un anticuerpo inmovilizado y se detecta a través de un marcaje, que puede ser de dos tipos, directo o indirecto. En el marcaje direc-

Tabla 1. Arrays de anticuerpos en el contexto de otras técnicas de proteomica

\begin{tabular}{lll}
\hline Caracteristicas & No Guiadas & Guiadas \\
\hline Proteínas medidas & Desconocido & Conocido \\
Objetivo & Identificación & Cuantificación \\
Ventajas & Descubrimiento & $\begin{array}{l}\text { Reproducibilidad, calibrable, } \\
\text { cuantificación precisa }\end{array}$ \\
Desventajas & $\begin{array}{l}\text { Resolución, reproducibilidad, sensibilidad, } \\
\text { automatización, cuantificación }\end{array}$ & \\
Tecnologías & $\begin{array}{l}\text { Geles bidimenos y protocolos optimizados } \\
\text { de masas } \\
\text { (SELDI, MALDI-TOF) }\end{array}$ & Arrays de tejido, \\
& & $\begin{array}{l}\text { Western Blotting múltiple } \\
\text { Arrays de proteína y anticuerpos }\end{array}$ \\
\hline
\end{tabular}


to, las proteínas son marcadas con un fluorocromo tipo cianinas (Cy3 o Cy5). En marcaje indirecto, las proteínas son marcadas a través de moléculas intermedias con distinta especificidad que más tarde serán detectadas por un anticuerpo marcado. La sensibilidad de este abordaje puede ser puede ser mejorada por el uso de protocolos de amplificación (Fig. 1).

Los arrays basados en el marcaje utilizando un solo anticuerpo permiten la incubación de dos muestras diferentes, cada una marcada con un fluorocromo distinto. Este tipo de arrays permite el uso de muestras control que son incubadas simultáneamente con la muestra a analizar facilitando la normalización posterior ${ }^{1-4}$. Otra ventaja es que estos ensayos son competitivos. Esto conduce a la mejora de la linearidad de la respuesta y el rango comparado con los ensayos nocompetitivos. La principal desventaja esta relacionada con la posible ruptura de la interacción del analito con el antígeno por el marcaje, la cual podría también limitar la detección, así como la sensibilidad y especificidad.
En el formato "sándwich", los anticuerpos inmovilizados capturan proteínas sin marcar, las cuales son detectadas por otros anticuerpos usando distintos métodos que generan la señal de detección. El uso de dos anticuerpos marcando cada proteína incrementa la especificidad comparado con los arrays de marcaje con un anticuerpo. El reducido ruido de fondo con estos ensayos también incrementa la sensibilidad. El formato "sándwich" sólo permite ensayos no competitivos, ya que sólo una muestra puede ser incubada sobre cada array ${ }^{1-3}$. Esto resulta en curvas sigmoidales para evaluar la unión del antígeno con el anticuerpo, comparado con la linealidad de los arrays competitivos. Además requieren curvas de calibración utilizando estándares de concentraciones conocidas. Los arrays de tipo "sándwich" son más difíciles de desarrollar de manera múltiple ya que es necesario disponer de pares de anticuerpos y antígenos purificados específicos para cada diana, que pudieran no estar disponibles. Además, la reactividad cruzada se incrementa

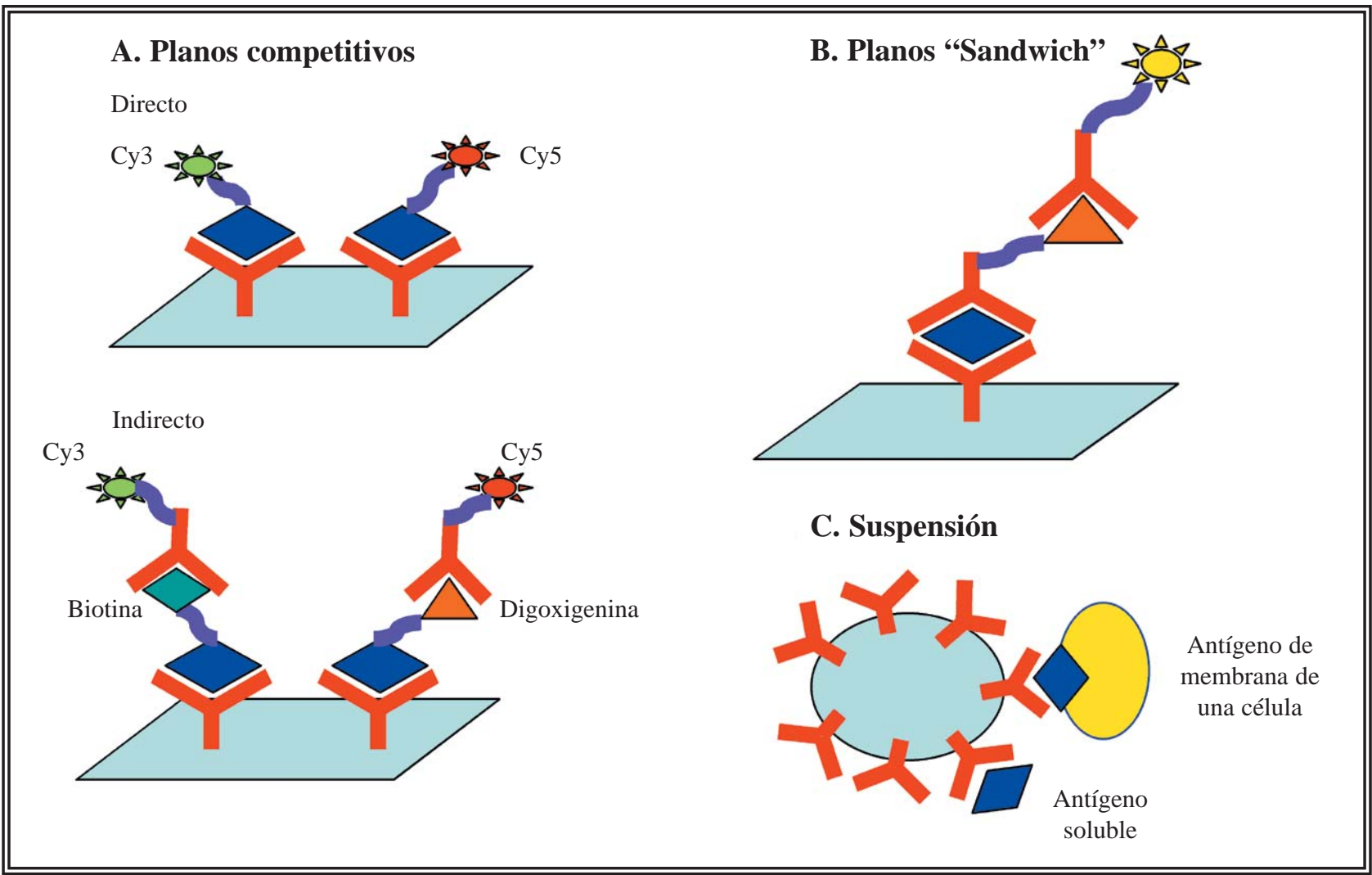

FIGURA 1. Formatos principales de arrays de anticuerpos planares y de suspensión. 
al ir incorporando un número mayor de proteínas a determinar. El tamaño práctico para realizar arrays "sándwich" múltiples está limitado entre 30-50 dianas diferentes. Esto contrasta con los arrays de un anticuerpo en los que solamente es la disponibilidad de anticuerpos y espacio disponible en el sustrato donde imprimir limita el número de proteínas potencialmente determinables.

Además de los arrays planos, los arrays en suspensión también utilizan diferentes fluorocromos revistiendo superficies circulares cubiertas con anticuerpos distintos, que son resolvibles espectralmente por sus distintas propiedades fluorescentes ${ }^{5,6}$. Al ser incubadas las superficies circulares con la muestra, se permite la unión de las proteínas con los anticuerpos de captura. Los anticuerpos detectores están marcados con fluorescencia permitiendo la detección de las proteínas a medir. El sistema separador de citometría de flujo permite evaluar con al menos dos tipos de laseres que leen el color o la identidad del haz de luz y la cantidad de anticuerpo fluorescente detector unido a la proteína diana ${ }^{5,6}$. Los arrays en suspensión multiparamétricos con sistemas de citometría de flujo representan un área tecnológica en expansión. Estos arrays permiten utilizar proteínas, autoantígenos o anticuerpos para medir multiparamétricamente proteínas y anticuerpos usando un sistema citométrico. Los avances en la instrumentalización probablemente harán este que este abordaje sea trasladable al campo clínico para la detección de células cancerigenas circulantes. En otra versión de este concepto, suspensiones celulares puede ser incubadas sobre arrays planos y el numero de células a las que se une el anticuerpo pueden ser cuantificadas por microscopia de campo oscuro. Estos arrays tienen el potencial de caracterizar múltiples proteínas de membrana en poblaciones celulares específicas o cambios en la superficie celular inducidos por terapias con drogas ${ }^{5,6}$

\section{APLICACIONES EN LA INVESTIGACIÓN EN CÁNCER}

Las aplicaciones clínicas de los arrays de anticuerpos varían desde el descubrimiento de marcadores tumorales para el diagnostico, pronóstico y respuesta terapéutica, a la caracterización de redes de señalización y modificaciones posttranslacionales de proteínas asociadas con tumorigénesis y progresión tumoral. Así, las aplicaciones de los arrays de anticuerpos pueden agruparse en aquellas que permiten determinar las bases moleculares de una enfermedad (proteómica funcional), las bases mecanísticas de acción y toxicidad farmacológicas (farmacoproteómica), y el desarrollo de nuevos marcadores específicos de la enfermedad (proteómica clínica) (Tabla 2).

Los arrays de anticuerpos ofrecen una gran variabilidad y versatilidad con respecto al tipo de muestras con las que pueden utilizarse, y las aplicaciones en la investigación en cáncer. Las muestras pueden variar desde sobrenadantes de líneas celulares cultivadas in vitro, extractos proteicos tisulares, fluidos biológicos como el suero, plasma, orina o el liquido cefalorraquídeo. Sin embargo, como muchos protocolos de marcaje e hibridación se suelen optimizar usando proteínas o péptidos purificados diluidos en un tampón matriz, no es aconsejable adoptar un método sin confirmar que sea el adecuado para el nuevo tipo

Tabla 2. Resumen de las aplicaciones principales descritas para los arrays de anticuerpos en el contexto de las áreas fundamentales de proteómica.

\begin{tabular}{ll}
\hline Áreas de proteómica & Aplicación \\
\hline Proteómica Funcional & $\begin{array}{l}\text { Caracterización de redes de señalización } \\
\text { implicadas en la progresión tumoral }\end{array}$ \\
& $\begin{array}{l}\text { Medida de cambios en modificaciones } \\
\text { post-translacionales en o niveles de expresión de } \\
\text { proteínas asociadas con la enfermedad }\end{array}$ \\
Farmacoproteómica & $\begin{array}{l}\text { Identificación de proteínas de unión en estudios } \\
\text { funcionales de desarrollo y descubrimiento de } \\
\text { dianas terapéuticas y fármacos antineoplásicos }\end{array}$ \\
& $\begin{array}{l}\text { Mapeo de epítopos para determinar regiones de } \\
\text { proteínas que unen a anticuerpos específicos }\end{array}$ \\
Proteómica clínica & $\begin{array}{l}\text { Descubrimiento y desarrollo de marcadores } \\
\text { tumorales específicos de la enfermedad }\end{array}$ \\
& $\begin{array}{l}\text { Caracterización de subtipos clínicos y pronóstico } \\
\text { de la evolución de la enfermedad }\end{array}$ \\
\hline
\end{tabular}


muestral, evaluando la posible existencia de interferencias que puedan dar lugar a una menor sensibilidad, mayor ruido de fondo, $u$ otros artefactos. El bloqueo o la pasivización de las superficies donde se imprimen los anticuerpos pueden modificarse y optimizarse dependiendo de la muestra a evaluación también. Los estudios de proteínas realizados en secreciones de fluidos biológicos o en sobrenadantes de cultivos celulares son técnicamente más sencillos que aquellos estudios realizados en extractos proteicos celulares, ya que en los fluidos biológicos todas las proteínas son solubles y el proceso preparativo de las muestras es relativamente sencillo. Los extractos proteicos celulares representan muestras más complejas y heterogéneas, que pudieran presentar más interferencias en los arrays de anticuerpos dando lugar a un mayor ruido de fondo. En general, los arrays de anticuerpos permiten estrategias técnicas precisas, factibles y reproducibles para medir múltiples proteínas simultáneamente en cualquier tejido, fluidos biológicos o especimenes de cultivos celulares de origen humano, ratón u otro organismo celular para realizar estudios de investigación en cáncer ${ }^{1-12}$.

La aplicabilidad de los arrays de anticuerpos a una gran variedad de muestras biológicas y clínicas amplia el abanico de usos innovadores de la tecnología. Los arrays de anticuerpos representan una plataforma proteómica versátil que permite el descubrimiento y la validación de nuevos marcadores tumorales específicos para cada tipo de neoplasia ${ }^{4,7}$. La medida multiparamétrica de proteínas permite un cribado precoz para el diagnostico, el pronostico y el establecimiento de marcadores predictivos de respuesta terapéutica a determinados antineoplásicos. Los arrays de anticuerpos han evolucionado como un intento de convertir los enzimoinmunoensayos habituales en la práctica clínica en mediciones multiparamétricas mediante un soporte miniaturizado. El concepto estándar de marcador individual de la enfermedad neoplásica puede así ser modificado por el uso de grupos de proteínas que estén asociadas con los estadios y/o evolución clínica o con las condiciones experimentales a evaluar. Es probable que el uso de mediciones proteicas combinadas a través de las tecnologías de arrays de anticuerpos mejorara la sensibilidad y la especificidad de los marcadores tumorales individuales existentes actualmente. En un futuro próximo, la medición eficiente de los perfiles proteicos específicos de cada tipo tumoral conducirá a una mejora en el manejo clínico del paciente afectado con cáncer y derivara en intervenciones terapéuticas más individualizadas a la agresividad de cada tumor.

Los arrays de anticuerpos pueden aplicarse para el estudio de ciertas interacciones proteínaproteína, proteína-ligando, actividades quinasa (fosforilación), y otras modificaciones post-translacionales de proteínas ${ }^{8-10}$. Estas aplicaciones requieren la disponibilidad de anticuerpos bien caracterizados que permitan distinguir por ejemplo, modificaciones post-translacionales originadas tras una terapia antineoplásica, o la monitorización de la sobre-expresión o el silenciamiento de una proteína. El uso de anticuerpos secundarios contra distintos epítopos de una proteína permite detectar cambios proteicos e interacciones asociadas con la biología o el diseño experimental a evaluar. La habilidad de los arrays de anticuerpos de monitorizar la información de estos niveles proteicos y cambios post-translacionales pone de manifiesto la relevancia de la proteómica funcional sobre los arrays genómicos. Más aun, la variedad de los formatos técnicos, y de las estrategias de diseño y detección permite estudios moleculares a nivel celular, incluso en determinados subcompartimentos celulares, usando muestras solubles o secretadas, y una gran variedad de aplicaciones. Estos análisis proporcionan una caracterización biológica de modificaciones moleculares y cambios de expresión de proteínas y redes de señalización críticas en los procesos de tumorigénesis y progresión tumoral.

\section{APLICACIONES EN EL ESTUDIO DEL CÁNCER VESICAL}

Los arrays de proteína y anticuerpo se han ido aplicando para el estudio de perfiles proteicos de extractos proteicos de tejidos y líneas celulares para detectar múltiples antígenos basándose en su unión al anticuerpo ${ }^{1-4}$. En línea con las capacidades de la tecnología, es posible usar esta estrategia para la detección e identificación masiva de biomarcadores usando muestras séricas humanas. Los perfiles proteicos medidos con arrays de proteína en suero han proporcionado una gran utilidad 
diagnóstica para distintas enfermedades ${ }^{1-10}$. Los arrays de anticuerpos se han testado también en enfermedad neoplásica urológica, como por ejemplo el cáncer prostático ${ }^{4}$. En el campo del estudio del cáncer vesical, también se ha evaluado la utilidad de estos perfiles proteicos en la detección de la enfermedad, seleccionando los anticuerpos en función de estudios de expresión génica comparando tumores vesicales con sus respectivos uroepitelios normales ${ }^{7}$. Mediante dos series independientes de arrays de anticuerpos específicos se pudieron discriminar a los pacientes con cáncer vesical de sujetos sanos así como de pacientes con patología urológica benigna y con otras neoplasias de distinta índole. Fue posible también clasificar a los pacientes con cáncer vesical en función de su supervivencia. No solamente se detectaron nuevas proteínas en suero, sino que se confirmó su origen en los tumores vesicales mediante inmunohistoquímica en arrays de tejidos con un gran número de tumores vesicales ${ }^{7}$. Este trabajo sostiene la especificidad y la utilidad clínica de los arrays de anticuerpos para el cáncer vesical apoyando: a) el diseño experimental seleccionando anticuerpos a partir de patrones genéticos característicos del tumor, b) la utilidad diagnóstica y pronóstica de la determinación de múltiples proteínas en suero simultáneamente. Sin embargo, aún no se han desarrollado algoritmos predictivos de respuesta terapéutica o selección del comportamiento clínico para identificar pacientes de alto riesgo en determinados estadios y procesos concretos en la progresión de la enfermedad. En el futuro podrán desarrollarse arrays de anticuerpos para evaluar si los productos de expresión de genes característicos de la progresión de la enfermedad en estadios avanzados identificados por estudios de expresión génica de tumores vesicales con arrays de ADN, pueden ser detectados en el suero de pacientes con cáncer vesical y servir para establecer y validad algoritmos pronósticos. Se espera que la detección de múltiples proteínas simultáneamente en suero lleve al descubrimiento de nuevos marcadores tumorales proteicos útiles para estratificar el pronóstico clínico de pacientes con cáncer vesical avanzado. Eventualmente, la tecnología podrá optimizarse y adaptarse a la medición multiparamétrica en muestras urinarias para la detección precoz y el seguimiento de la enfermedad.

\section{RETOS POR RESOLVER EN LA UTILIZACIÓN DE ARRAYS DE ANTICUERPOS}

Los arrays de anticuerpos siguen estando en fase de desarrollo y optimización. Entre los múltiples retos que pueden mejorarse en su diseño y aplicaciones pueden citarse: a) el desconocimiento de los procesos químicos asociados a la inmovilización de proteínas; b) los rangos dinámicos limitados a 2 o tres ordenes de magnitud; c) obtener exactitud y reproducibilidad similares a los enzimoinmunoensayos clínicos; d) la complejidad molecular de las proteínas y su desnaturalización que puede afectar a su inmunoreactividad; e) falta de estándares y calibradores para todos los anticuerpos; f) el desarrollo de anticuerpos de alta afinidad específicos para los antígenos a cuantificar $^{1-4}$. Tales retos se están analizando en esfuerzos multicéntricos a nivel internacional en la organización "Human Proteome Organization (HUPO)" con los objetivos de estandarizar las mediciones clínicas en suero y plasma en los estudios con técnicas de proteómica. Los estudios iniciales aportan guías en variables analíticas que pueden alterar el análisis de muestras derivadas de la sangre, incluyendo la selección del tipo muestral y su estabilidad para cada tecnología, el uso de inhibidores proteicos y la estandarización clínica ${ }^{11}$. Como parte del abordaje de esta organización, es también critico estandarizar las estrategias estadísticas para conseguir alta fiabilidad en los procesos de identificación proteica y los análisis de datos proteómicos. Estos esfuerzos y estrategias enfocados en la integración de las bases de datos de proteómica podrán conducir a un conocimiento preciso y global del proteoma humano ${ }^{12}$.

\section{CONCLUSIONES}

Las aplicaciones de los arrays de anticuerpos están aumentando en sus objetivos y efectividad clínica y biológica. El uso de distintas metodologías proteómicas simultáneamente no sólo aporta información complementaria sino que también resulta en un beneficio adicional en el diagnostico del cáncer y la caracterización de la biología asociada a la progresión tumoral. Es esperable que el desarrollo de nuevos formatos de arrays de anticuerpos aceleren los procesos de descubrimiento de marcadores tumorales específicos para 
cada enfermedad así como la caracterización de dianas terapéuticas específicas para cada tumor. Estos avances darán lugar al establecimiento y validación de marcadores de pronóstico clínico y de respuesta terapéutica que constituirán guías clave para el desarrollo de terapias individualizadas que tengan en consideración la agresividad específica de cada tumor.

\section{REFERENCIAS}

1. Haab BB, Dunham MJ, Brown PO. Protein microarrays for highly parallel detection and quantitation of specific proteins and antibodies in complex solutions. Genome Biol. 2001;2:(2):RESEARCH0O04.

2. Sanchez-Carbayo M. Antibody arrays: technical considerations and clinical applications in cancer. Clin Chem. 2006; 52(9): 1651-1659.

3. Kingsmore SF. Multiplexed protein measurement: technologies and applications of protein and antibody arrays. Nat Rev Drug Discov. 2006;5(4):310-320.

4. Miller JC, Zhou H, Kwekel J, Cavallo R, Burke J, Butler EB, Teh BS et al. Antibody microarray profiling of human prostate cancer sera: antibody screening and identification of potential biomarkers. Proteomics. 2003;3(1):56-63.

5. Pang S, Smith J, Onley D, Reeve J, Walker M, Foy C. A comparability study of the emerging protein array platforms with established ELISA procedures. J Immunol Meth. 2005; 302:(1-2):1-12.

6. De Jager W, Rijkers GT. Solid-phase and bead-based cytokine immunoassay: a comparison. Methods 2006;38(4):294303.
7. Sanchez-Carbayo M, Socci ND, Lozano JJ, Haab BB, Cordon-Cardo C. Profiling bladder cancer using targeted antibody arrays. Am J Pathol. 2006;168(1):93-103.

8. Nielsen UB, Cardone MH, Sinskey AJ, MacBeath G, Sorger PK. Profiling receptor tyrosine kinase activation by using Ab microarrays. Proc Natl Acad Sci. U S A. 2003;100(16): 9330-9335.

9. Gembitsky DS, Lawlor K, Jacovina A, Yaneva M, Tempst P. A prototype antibody microarray platform to monitor changes in protein tyrosine phosphorylation. Mol Cell Proteomics. 2004;3(11):1102-1118.

10. Ivanov SS, Chung AS, Yuan ZL, Guan YJ, Sachs KV, Reichner JS et al. Antibodies immobilized as arrays to profile protein post-translational modifications in mammalian cells. Mol Cell Proteomics. 2004;3(8):788-795.

11. Rai AJ, Gelfand CA, Haywood BC, Warunek DJ, Yi J, Schuchard MD et al. HUPO Plasma Proteome Project specimen collection and handling: towards the standardization of parameters for plasma proteome samples. Proteomics. 2005;5(1):3262-3277.

12. States DJ, Omenn GS, Blackwell TW, Fermin D, Eng J, Speicher DW et al. Challenges in deriving high-confidence protein identifications from data gathered by a HUPO plasma proteome collaborative study. Nat Biotechnol. 2006;24 (3):333-338.

Correspondencia autora: Dra. M. Sánchez-Carbayo Grupo de Marcadores Tumorales, 208A

Centro Nacional de Investigaciones Oncológicas Melchor Fernández Almagro 3. 28029 Madrid. Tel.: +34917 328053

E-mail autora: mscarbayo@cnio.es

Información artículo: Original - Nuevas tecnologías 